\section{Mehr Lebensqualität bei Kopf-Hals-Tumoren}

Bei der Therapie inoperabler, lokal fortgeschrittener Kopf-Hals-Tumoren ist die Lebensqualität als Therapieziel von großer Bedeutung. In einer Studie verglich man diesen Parameter bei zwei Induktionsregimes.

n der Phase-III-Studie EORTC 24971/

TAX 323 zeigte die Zugabe von Docetaxel zu einer Cisplatin/5-FluorouracilInduktionschemotherapie (TPF) ein längeres progressionsfreies und Gesamtüberleben in dieser Situation bei weniger Toxizität als PF allein.

In der Studie hatten 358 Patienten mit nicht resektablen lokoregionär fortgeschrittenen Plattenepithelkarzinomen bis zu vier Zyklen der jeweiligen Chemotherapie erhalten. Zu Beginn, nach dem zweiten Zyklus sowie vier, sechs und neun Monate nach der anschließenden Strahlentherapie wurden mit Hilfe des QLQC30- Fragebogens und des für KopfHals-Tumoren spezifischen Lebensqualitätsfragebogens EORTC QLQ-H\&N35 auch Daten zur Lebensqualität erfasst. Hatten zu Beginn der Studie noch 97 \% der Patenten Angaben zur Lebensqualität gemacht, lag die Compliance mit dem Fragebogen nach sechs Monaten nur noch bei $54 \%$, was die Aussagekraft der Ergebnisse beeinträchtigt.

Über den Beobachtungszeitraum von sechs Monaten zeigte sich ein Trend hin zu einer insgesamt verbesserten Lebensqualität. Sechs Monate nach Bestrahlung lag der globale gesundheitsbezogene Lebensqualitätsindex im TPF-Arm klar über dem im PF-Arm, die hohe Zahl an fehlenden Angaben lässt aber keine endgültige Aussage zu. Die Kopf-Hals-Tumorspezifische Lebensqualität zeigte mögliche Vorteile von TPF gegenüber PF insbeson- dere bei den Aspekten Schlucken und Husten nach dem zweiten Zyklus: In beiden Bereichen nahmen die Symptome bei TPF-Therapie im Median stärker ab als bei Behandlung mit PF allein.

Fazit: Eine Induktionschemotherapie mit TPF vor der Radiatio von inoperablen, lokal fortgeschrittenen Kopf-Hals-Tumoren bietet möglicherweise nicht nur einen Vorteil hinsichtlich progressionsfreiem und Gesamtüberleben bei verringerter Toxizität im Vergleich zu einer Induktion mit PF. Die Auswertung der Lebensqualitätsdaten lässt hoffen, dass so auch die Lebensqualität über längere Zeit günstig beeinflusst werden kann. Friederike Klein

Van Herpen CML et al. Short-term health-related quality of life and symptom control with docetaxel, cisplatin, 5-fluorouracil (TPF) and cisplatin, 5-fluorouracil (PF) for induction in unresectable locoregionally advanced head and neck cancer patients (EORTC 24971/TAX 323). Br J Cancer 2010;103: 1173-81

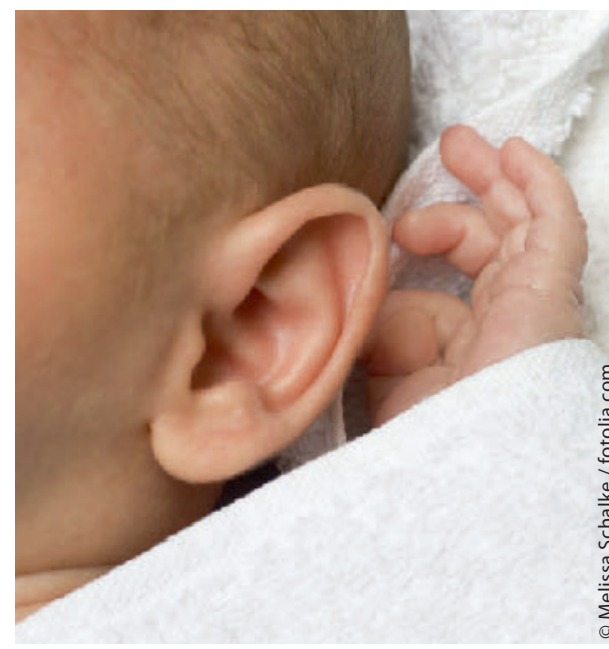

Aufmerksam lauschen und gut hören zu können ist eine der wichtigsten Voraussetzungen für die Sprachentwicklung. bekannten Risikofaktoren folgreich absolviert hatten. Damit waren diese Kinder zum Zeitpunkt der Diagnose und einer möglichen Operation bereits älter.

Fazit: Bei einem Teil der Kindern scheitert die frühe Diagnose durch einen späteren Beginn des sensoneuralen Hörverlustes. Dieses Problem, so stellen die Autoren fest, kann durch die gegenwär- tige Form des Hörscreenings nicht behoben werden.

Dr. Christine Starostzik

Young $\mathrm{N}$ M et al. Limitations of Universal Newborn Hearing Screening in Early Identifidates. Arch Laryngol Head Neck Surg. 2011; 137: 230-234. cation of Pediatric Cochlear Implantat Candi- 